\title{
Moving capability of mechanism based on topological graph theory
}

\author{
Jian-Guo Luo ${ }^{1, \mathrm{a}, \dagger}$ and Mao-Yan $\mathrm{He}^{2, \mathrm{~b}}$ \\ ${ }^{I}$ Mechanical and Electrical Engineering Department, \\ North China Institute of Science and Technology, \\ East Surbub of Beijing, China, 101601 \\ ${ }^{2}$ Labour Union, North China Institute of Science and Technology, \\ East Surbub of Beijing, China, 101601 \\ aluojg_1598@126.com, bhmy666@ncist.edu.cn
}

\begin{abstract}
Based on the analysis of current developing state of graph theory, define the description of spacial moving capability of common couples and translation base and rotation base of mechanism, based on the new description method in topological graph theory. DOF(degree of freedom) of hybrid mechanism analyzed with example based on the definition of dimensionality of branch spacial moving capability and mechanism spacial moving capability, necessary and sufficient condition of non singularity of mechanism presented, as well as the necessary and sufficient condition of singularity of mechanism deduced, in-phase and assimilation condition and in-phase and dissimilarity condition and asynchronism condition of limitation of input base of branch adopted, case number of position singularity and pose singularity and position and pose singularity obtained then, still the way of founding the combination and case number of common serial mechanism and parallel mechanism and hybrid mechanism mentioned.
\end{abstract}

Keywords: Mechanism; Graph theory; Set; Degree of freedom; Singularity

\section{Introduction}

Research on the kinematic chain has been more than one century, in 1964, Freudenstein and Dobrjanskyj [1] introduced graph theory into the mechanics for the first time, which used to represent the topological structure of kinematic chain. A large variety of components and couples in kinematic chains have been integrated out by making use of graph theory [2], these kinematic chains can also be translated into more different mechanisms, which greatly enriched the structure type of mechanism, the introduction of graph theory has brought great convenience to study the topological structure of the kinematic chains, and new way opened up for the study of mechanics. Meanwhile, the topological map can be described by matrix such as adjacent matrix, correlation matrix, etc. [3], matrix can be used for mathematical analysis easily, so the graph theory provide to a powerful mathematical tool for the study of mechanism structure, which greatly 
accelerated the concept design of auto machine based on computer, thus forming a new direction of research on the basic theory of mechanism.

Basic understanding of mechanism is to know its degree of freedom (DOF). Calculation of degree of freedom originally is a simple problem, correct results can be obtained by making use of the traditional formula of Kutzbach-Grübler [4-6], which based on arithmetic only. However, in the course of development of mechanics, some mechanisms founded not meet the above mentioned formula [7-8], Huang applied theory of screw to deal with this problem, proposed constrained screw to found the DOF [9]. However, only abstract analysis on DOF can be done by this method, this paper presents a new approach based on graph theory, both intuitive analysis on DOF and analysis on singularity may be occur can be done by using that, which also have important significance in the solution of working space of mechanism.

\section{Analysis on DOF of Mechanism}

According to description methods in reference [10] to [13], the movement of mechanism can be decomposed into translation along the determined axis and rotation around the determined axis, as mentioned earlier, which defined as kinematic base, namely translation base and rotation base. Therefore, the spatial moving capability of mechanism can be expressed by the set of spatial moving capability of kinematic base synthetically, reference [13] present an example, refer to this method, analysis on DOF of hybrid mechanism show in Fig. 1.

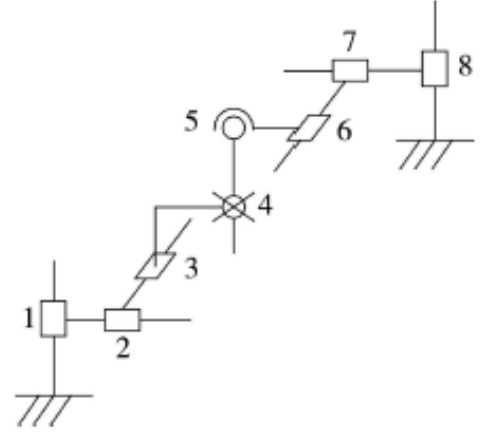

Figure 1: Diagram of hybrid mechanism

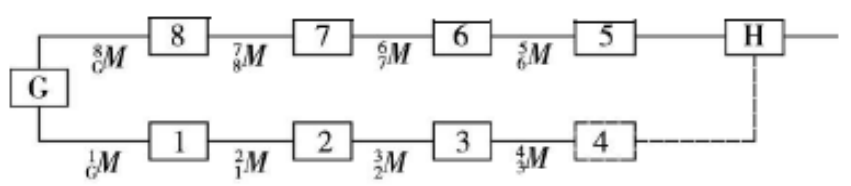

Figure 2: Graph theory of mechanism in Fig.1 
Making use of the description show in Fig. 2, there are two kinematic chains named as branch chain 1 and branch chain 2, which consist of components $\mathrm{G}$, $1,2,3,4$ and components $G, 8,7,6,5$ respectively, the spatial moving capability $M_{1}$ and $M_{2}$ of branch chain 1 and branch chain 2 can be expressed respectively as

$$
\begin{aligned}
M_{1}= & K_{1}\left\{t_{1} \mid t_{1} \in\left(\operatorname{trans}\left(n_{1}\right) \cup \operatorname{trans}\left(n_{2}\right) \cup\right.\right. \\
& \left.\left.\operatorname{trans}\left(n_{3}\right) \cup \operatorname{rot}\left(n_{2}\right) \cup \operatorname{rot}\left(n_{3}\right)\right)\right\} \\
M_{2}= & K_{2}\left\{t_{2} \mid t_{2} \in\left(\operatorname{trans}\left(n_{1}\right) \cup \operatorname{trans}\left(n_{2}\right) \cup\right.\right. \\
& \left.\left.\operatorname{trans}\left(n_{3}\right) \cup \operatorname{rot}\left(n_{1}\right) \cup \operatorname{rot}\left(n_{2}\right) \cup \operatorname{rot}\left(n_{3}\right)\right)\right\},
\end{aligned}
$$

(1) Dimensionality of spatial moving capability of branch chain. $\operatorname{Dim}\left(M_{1} \mid K_{1}\right)$ defined as set of spatial moving capability of branch chain 1 in domain $K_{1}$, sum of the number of axis of linear independent in subset of translation $M_{1-\mathrm{P}}=K_{3}\left\{t_{3} \mid t_{3} \in\left(\operatorname{trans}\left(n_{1}\right) \cup \operatorname{trans}\left(n_{2}\right) \cup \operatorname{trans}\left(n_{3}\right)\right)\right\}$ and the number of axis of linear independent in subset of rotation $M_{1-\mathrm{R}}=K_{4}\left\{t_{4} \mid t_{4} \in\left(\operatorname{rot}\left(n_{2}\right) \cup \operatorname{rot}\left(n_{3}\right)\right)\right\}$ is the dimensionality of spatial moving capability of branch chain 1. Similarly, $\operatorname{Dim}\left(M_{2} \mid K_{2}\right)$ defined as set of spatial moving capability of branch chain 2 in domain $K_{2}$, among them, $M_{1}=M_{1-\mathrm{P}} \cup M_{1-\mathrm{R}}, \operatorname{Dim}\left(M_{1} \mid K_{1}\right)=5$ and $\operatorname{Dim}\left(M_{2} \mid K_{2}\right)=6$ can be obtained from Figure 2 and expression 1 to 2 .

(2) Dimensionality of spatial moving capability of mechanism. $\operatorname{Dim}\left(M_{3} \mid K_{3}\right)$ defined as set of spatial moving capability of mechanism in domain $K_{3}$, sum of the number of axis of linear independent in subset of translation $M_{3-\mathrm{P}}=K_{4}\left\{t_{4} \mid t_{4} \in\left(\operatorname{trans}\left(n_{1}\right) \cup \operatorname{trans}\left(n_{2}\right) \cup \operatorname{trans}\left(n_{3}\right)\right)\right\}$ and the number of axis of linear independent in subset of rotation $M_{3-\mathrm{R}}=K_{5}\left\{t_{5} \mid t_{5} \in\left(\operatorname{rot}\left(n_{1}\right) \cup \operatorname{rot}\left(n_{2}\right) \cup \operatorname{rot}\left(n_{3}\right)\right)\right\}$ is the dimensionality of spatial moving capability of mechanism. there

$$
\begin{aligned}
M_{3}= & M_{1} \cup M_{2}=K_{3}\left\{t_{3} \mid t_{3} \in\left(\operatorname{trans}\left(n_{1}\right) \cup \operatorname{trans}\left(n_{2}\right) \cup\right.\right. \\
& \left.\left.\operatorname{trans}\left(n_{3}\right) \cup \operatorname{rot}\left(n_{1}\right) \cup \operatorname{rot}\left(n_{2}\right) \cup \operatorname{rot}\left(n_{3}\right)\right)\right\},
\end{aligned}
$$

$\operatorname{Dim}\left(M_{3} \mid K_{3}\right)=6$ can be obtained then, the DOF of hybrid mechanism show in Fig. 1 is 6 consequently.

The mechanism have the spatial moving capability provided by branch chain1 and branch chain 2, and the necessary and sufficient condition for no reduce in dimensionality is $\operatorname{Dim}\left(M_{1} \mid K_{1}\right)=\operatorname{Dim}\left(M_{1} \mid K_{3}\right)$ and 
$\operatorname{Dim}\left(M_{2} \mid K_{2}\right)=\operatorname{Dim}\left(M_{2} \mid K_{3}\right)$, this proposition can be proved by reductio ad absurdum quickly, it omitted here.

\section{Analysis on Singularity of Mechanism}

When singularity occurred, the number of DOF will reduce, inconsistent exists between the dimensionality of branch chain in domain of itself and the dimensionality of branch chain in domain of mechanism, take the above mentioned hybrid mechanism as an example, there will

$$
\operatorname{Dim}\left(M_{1} \mid K_{1}\right)>\operatorname{Dim}\left(M_{1} \mid K_{3}\right)
$$

Or/and

$$
\operatorname{Dim}\left(M_{2} \mid K_{2}\right)>\operatorname{Dim}\left(M_{2} \mid K_{3}\right) .
$$

The same result exists for parallel mechanism, there will no such result for serial mechanism.

The occurrence of singularity of hybrid mechanism show inn Fig.1 will bring the limit or assimilation of input translation base on branch chain 1 or/and branch chain 2.

(1) Position singularity. numbers of in-phase and assimilation case of limitation of input base of two branch chains are

$$
\mathrm{S}_{1}=\mathrm{C}_{3}^{1}+\mathrm{C}_{3}^{2}+\mathrm{C}_{3}^{3}=7
$$

The number of position singularity is $\mathrm{S}_{1}=7$.

(2) Pose singularity. numbers of in-phase and dissimilarity case of limitation of input base of two branch chains are

$$
S_{2}=2 C_{3}^{1}+2\left(C_{3}^{1} C_{2}^{1}+C_{3}^{3}\right)+2\left(C_{3}^{3}+C_{3}^{2}+C_{3}^{1}\right)=38
$$

and numbers of asynchronism case of limitation of input base of two branch chains are

$$
S_{3}=2\left(C_{3}^{1}+C_{3}^{2}+C_{3}^{3}\right)=14
$$
8.

the number of pose singularity is $\mathrm{S}_{2}+\mathrm{S}_{3}=38+14=52$ from express. 7 to

(3) position and pose singularity. The number of position limitation of each branch chain minus the number of position singularity and the number of pose singularity of mechanism, the number of position and pose singularity of mechanism can be obtained as following 


$$
\begin{gathered}
\mathrm{S}_{0}=2\left(\sum_{i=1}^{3} \mathrm{C}_{3}^{i}\right)\left(\sum_{j=1}^{3} \mathrm{C}_{3}^{j}\right)=98 \\
\mathrm{~S}_{4}=\mathrm{S}_{0}-\mathrm{S}_{1}-\left(\mathrm{S}_{2}+\mathrm{S}_{3}\right)=98-7-52=39
\end{gathered}
$$

From the above, the number of position or/and pose singularity can be easily calculated, this method can also be suitable for parallel mechanism with symmetrical structure, for common serial mechanism and parallel mechanism and hybrid mechanism, we can make use of the method of combination of reducing number of translation base and rotation base on branch chain of mechanism, that part will be described in detail in subsequent articles.

\section{Conclusion}

New method of graph theory on mechanism proposed based on the study on traditional graph theory of mechanism, conclusions obtained as following.

(1) description in graph theory on the spatial relative moving capability between adjacent components, it will be valid in the research on serial mechanism, parallel mechanism and hybrid mechanism.

(2) dimensionality of spatial moving capability of branch chain and mechanism defined based on the graph theory and kinematic base, solving method on DOF and necessary and sufficient condition of non singularity of mechanism deduced then.

(3) making use of criterion of inconsistent of dimensionality of spatial moving capability of branch chain in the domain of branch chain and domain of mechanism, in-phase and assimilation case and in-phase and dissimilarity case and asynchronism case of limitation of input base of branch adopted, case number of position singularity and pose singularity and position and pose singularity obtained through the method of combination of reducing number of translation base and rotation base on branch chain of mechanism.

\section{Acknowledgement}

This research was financially supported by the HeBei Province Science and Technology Foundation (No.Z2015072, No.15275508) and NCIST Foundation (No. 3142015023).

\section{References}

[1] F. Freudenstein, L. Dobrjanskyj: submitted to Congress of Applied Mechanics (1964)

[2] K. D. Lu, Graph theory and its application, Tsinghua University Publishing House, Beijing, 1984. 
[3] H. F. Ding, Z. Huang: submitted to Journal of Mechanical Engineering (2007)

[4] M. Grübler, Allgemeine Eigenschaften der zwangläufigen ebenen kinematische, Civilingenieur, Verhandlungen, 1883.

[5] M. Grübler, Allgemeine Eigenschaften der zwangläufigen ebenen kinematische, Civilingenieur, Verhandlungen, 1885.

[6] K. Kutzbach, Einzelfragen aus dem gebiet der Maschinenteile, Zeitschrift der Verein Destscher, Ingenieur, 1933.

[7] C. H. Suh, C. W. Radcliffe, Kinematics and Mechanisms Design, Prentice Hall, New York, 1978.

[8] G. N. Sandor, A. G. Erdman, Advance Mechanism: Analysis and Synthesis, Prentice Hall, New York, 1984.

[9] Z. Huang, Y. S. Zhao and T. S. Zhao, High level spacial mechanics, High Education Publishing House, Beijing, 2006.

[10] T. L. Yang, Basic theory on configuration and kinematics and kinetics of machinery system, Machinery Industry Publishing House, Beijing, 1995.

[11] T. S. Mruthyunjaya: submitted to Journal of Mechnism and Machine Theory (2003)

[12] Z. Y. Chang, C. Zhang and Y. H. Yang: submitted to Journal of Mechnism and Machine Theory (2002).

[13] J. G. Luo, M. Y. He and Z. Lu: submitted to Journal of Mechanical Engineering (2008) 